\title{
THE EPIDEMIOLOGY OF ZOONOTIC PARASITES OF FARIM ANIMALS AND BIRDS IN CHINA IN THE PAST TEN YEARS
}

\author{
Kun $\mathrm{Li}^{1,2^{*}}$, Yaping Wang ${ }^{3}$, Aoyun $\mathrm{Li}^{3}$, Muhammad Fakhar-e-Alam Kulyar ${ }^{3}$ and Zeeshan Ahmad Bhutta ${ }^{4}$
}

${ }^{1}$ Institute of Traditional Chinese Veterinary Medicine, College of Veterinary Medicine, Nanjing Agricultural University, Nanjing 210095, China; ${ }^{2}$ MOE Joint International Research Laboratory of Animal Health and Food Safety, College of Veterinary Medicine, Nanjing Agricultural University, Nanjing 210095, PR China; ${ }^{3}$ College of Veterinary Medicine, Huazhong Agricultural University, Wuhan 430070, China; ${ }^{4}$ The Royal (Dick) School of Veterinary Studies, University of Edinburgh, Easter Bush Campus, Midlothian, EH25 9RG, Scotland, United Kingdom

*Corresponding author: lik2014@ sina.com

\section{INTRODUCTION}

\section{Toxoplasmosis}

In 1908, Nicolle and Manceaux first discovered Toxoplasma gondii in Africa (Ferguson and Henriquez 2005). Since then, people have recognized Toxoplasmosis. Toxoplasmosis is a worldwide zoonotic parasitic disease, caused by Toxoplasma gondii of the genus Toxoplasma (Hernández-de-Los-Ríos et al. 2019). This intracellular parasite can infect humans and almost all warm-blooded animals, including mammals and birds. According to an estimate, about one-third of the world's population is infected with Toxoplasma gondii (Robert-Gangneux and Dardé 2012).

\section{Morphology}

Toxoplasma gondii is an intracellular parasite, divided into five forms according to its developmental stages: (1) Tachyzoites, which rapidly divide into nucleated cells and occupy the entire cytoplasm of cells of the host, and called as pseudocysts (Fig. 1). (2) Cysts which slowly proliferate in the cyst wall secreted by the worm, called a cyst, which contains hundreds of bradyzoites (Dubey et al. 1998). (3) Schizont comprises bradyzoites or sporozoites, etc. Schistosomes proliferate in cat small intestinal epithelial cells to form a collection of merozoites. (4) Gametophytes are large gametes (female) and small gametes (male), which form zygotes after fertilization and finally develop into oocysts. (5) Oocysts refer to the inside of oocysts.

A sporophyte develops and reproduces, forming 2 sporangia, and then each sporangium differentiates into 4 sporozoites. Toxoplasma gondii develops in two stages, namely the extra-intestinal mucosa stage and the intraintestinal mucosa stage. The former develops in various intermediate hosts and main tissue cells of end-staying infectious diseases. The latter only develops in the epithelial cells of the small intestinal mucosa of the last host (Aguirre et al. 2019).

\section{Life style}

The life history of Toxoplasma gondii comprises two stages, divided into sexual reproduction and asexual reproduction.
Sexual reproduction only occurs in cats (Robert-Gangneux et al. 2012). When cats eat meat containing cysts, the cyst wall is destroyed by gastric acid, and the bradyzoites in the cyst are released and settle in the intestinal epithelial cells. Then it undergoes sexual development, forming male and female gametes (gamete reproduction) (Ferguson 2002). After fertilization, the oocysts enter the intestine and are excreted with feces (Fig. 2).

When the intermediate host eats food or drinks water contaminated by oocysts, the oocysts will enter the digestive tract and rupture. Resultantly, it spreads through the intestinal wall with the blood to the tissue of the entire body and proliferates in the cells through budding. The host's immunity can slow down the reproduction of the protozoa and form a cyst wall, which becomes a cyst. Cysts are most commonly associated with the brain and skeletal muscles and can survive for a long time (Ferguson and Hutchison 1987).

\section{Epidemiology}

Toxoplasmosis is one of the most common infectious diseases in mammals, with a worldwide distribution (Mohammed et al. 2019). According to the survey, the incidence rate in developing countries is higher than that in developed countries, and the incidence is higher in some backward regions, such as Latin America, the Middle East, and Africa (Montoya and Liesenfeld 2004). Diseased animals and insect-carrying animals are the sources of infection of Toxoplasma gondii. Cats are the last host of this parasite. Oral infection is the fundamental way of transmission of the disease. Humans and different carnivores are infected by eating raw or undercooked meat contaminated by encapsulated parasites. At the same time, Toxoplasma gondii can be transmitted to offspring through the placenta. Table 1 shows the factors that trigger the Toxoplasma outbreak.

\section{Pathogenesis}

The pathogenic effects of Toxoplasma gondii depend upon virulence of the parasite and immune status of the host (Zhang et al. 2018). The tachyzoite stage is the primary treatment stage of Toxoplasma gondii. After the worms invade cells of the body, they proliferate in an enormous 
Table 1: As of 2018, a summary of factors that triggered the outbreak of Toxoplasma gondii. Data referenced from a previous research (Pinto-Ferreira et al. 2019).

\begin{tabular}{lc}
\hline Related factors & Incidence (\%) \\
\hline Contaminated meat & 47.1 \\
Oocysts & 44.1 \\
Intake of oocysts in water & 20.6 \\
Contact with sand and soil & 17.6 \\
Consumption of vegetables & 5.9 \\
Tachyzoites in raw milk & 8.8 \\
\hline
\end{tabular}

amount, destroying cells of the body. At the same time, Toxoplasma gondii produces toxins, causing inflammation in the body (Burger et al. 2018). Chronozoites in the cyst can cause chronic infection. The continuous proliferation of the chronic zoites leads to a larger size, which can squeeze the organs and impair their functions.

\section{Symptoms}

The common clinical symptom is head and neck lymph node disease, but inguinal lymph node, retroperitoneal lymph node, and mesenteric lymph node disease may also occur. Usually, swollen nodules of $0.5-3.0 \mathrm{~cm}$ diameter appear in the lymph nodes (Montoya and Liesenfeld, 2004). It is also accompanied by symptoms such as fever, malaise, sore throat, and rash. In a host with strong immunity, chronic infection is usually asymptomatic. In contrast, in chronically infected people with immunodeficiency, the brachyzoites in the body transform into actively replicating tachyzoites, leading to necrotizing encephalitis and even death.

\section{Diagnosis}

According to the patient's immune background, disease status and clinical signs, the diagnosis can be made through pathogenic examination and serological examination. Pathogen inspection includes examination of body fluids or tissues for worms through a microscope. For direct necropsy of animals, tissues such as lungs and liver are stained for microscopic examination. For serological examination, IHA and ELISA methods are often used at present. The analysis should be carried out 2-4 weeks after the first examination because there is possibility of false positive results. If the IgA antibody titer increases by over 4 times, it is taken as positive.

\section{Trichinosis}

Trichinosis is a zoonotic disease caused by Trichinella spiralis, a parasite of the genus Trichinellaceae. Besides humans, animals including pigs, dogs, cats, rodents and wild boars can be infected with this parasite (Arefkhah et al. 2020). Trichinella spiralis is a common pathogen that causes human diseases and can cause infection after eating raw or undercooked meat containing Trichinella spiralis larvae (Dimzas et al. 2019). There are many clinical symptoms, including general fever, abdominal pain, diarrhoea, nausea, vomiting or muscle pain, and symptoms of myocarditis and encephalitis (Rawla and Sharma 2021).
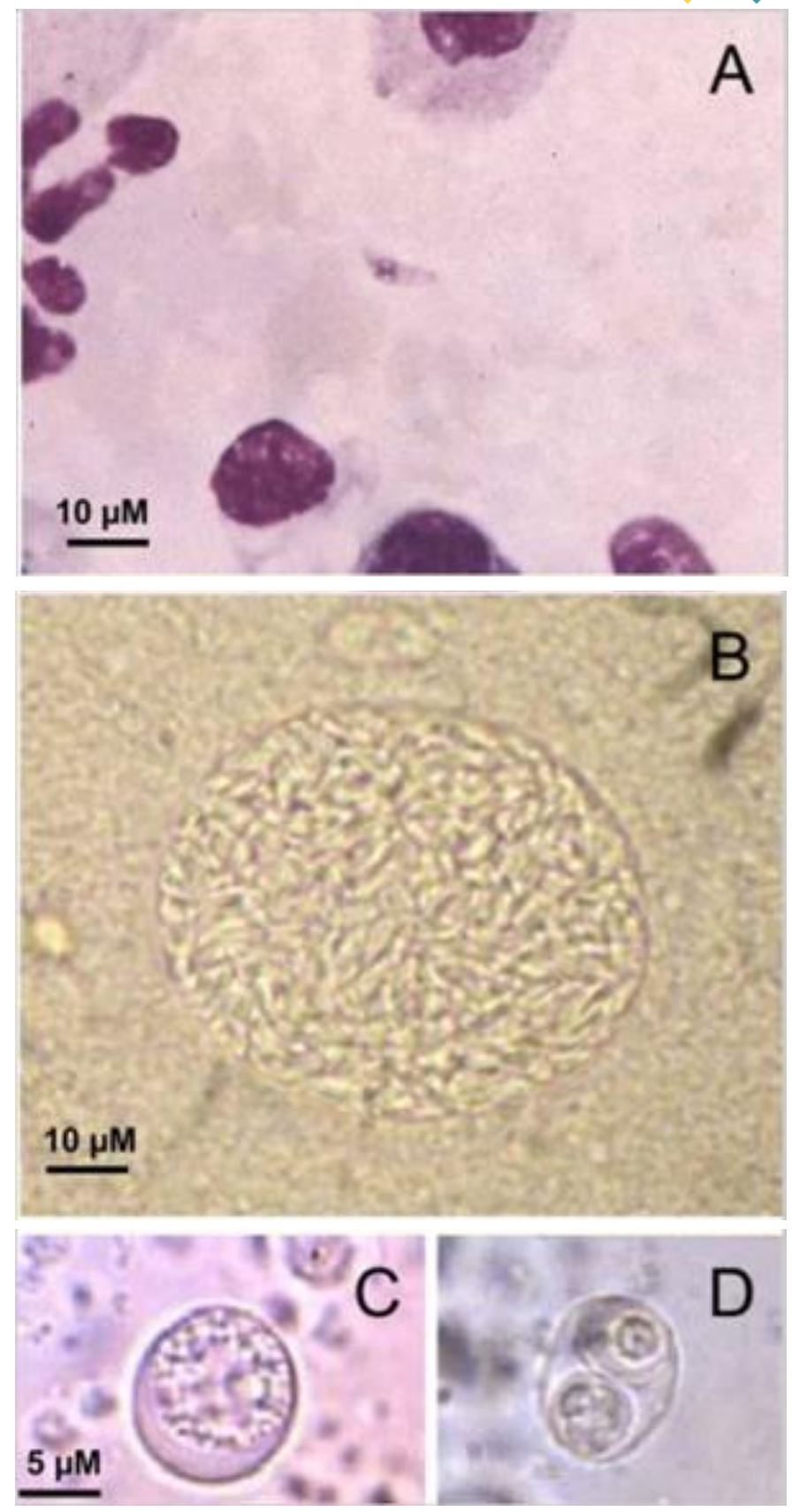

Fig. 1: Biological stages of Toxoplasma gondii: Microscopic examination of tachyzoites in a bronchoalveolar lavage fluid sample stained with Giemsa (A) (magnification, $\times 500$ ); a cyst in the brain of an infected mouse (B) (magnification, $\times 500$ ); unsporulated (C) and sporulated (D) oocysts (magnification, $\times 1,000$ ). (Robert-Gangneux et al. 2012).

\section{Morphology}

Adult Trichinella spiralis are small and dioecious. The average length of adult males is $1.2 \mathrm{~mm}$, and that of females is $2.2 \mathrm{~mm}$ (Peters and Pasvol 2007). The front part of the worm is thinner, the oesophagus; the back part is thicker, containing the intestines and reproductive organs. The larvae are located in the cyst and are coiled in a spiral shape. The fully developed larva usually has 2.5 discs. The cyst is fusiform, with its long axis parallel to the muscle fibres, and has two walls. Generally, there is only one larva, but some may have 6-7 (Fig. 3). 


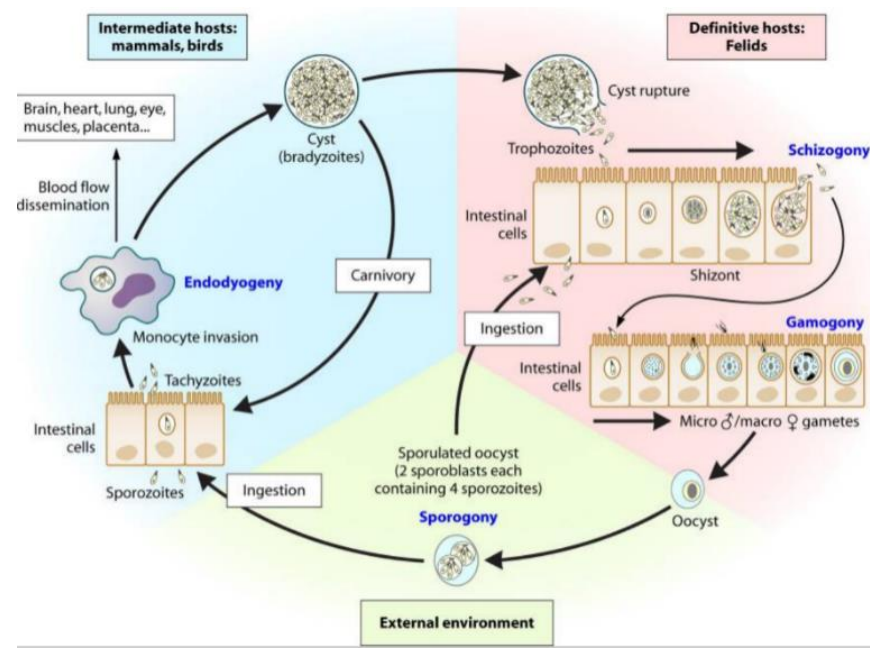

Fig. 2: Life cycle of Toxoplasma gondii. Figure shows the biology, infection, and replication of three infective stages of parasites in their respective hosts (Robert-Gangneux et al. 2012).

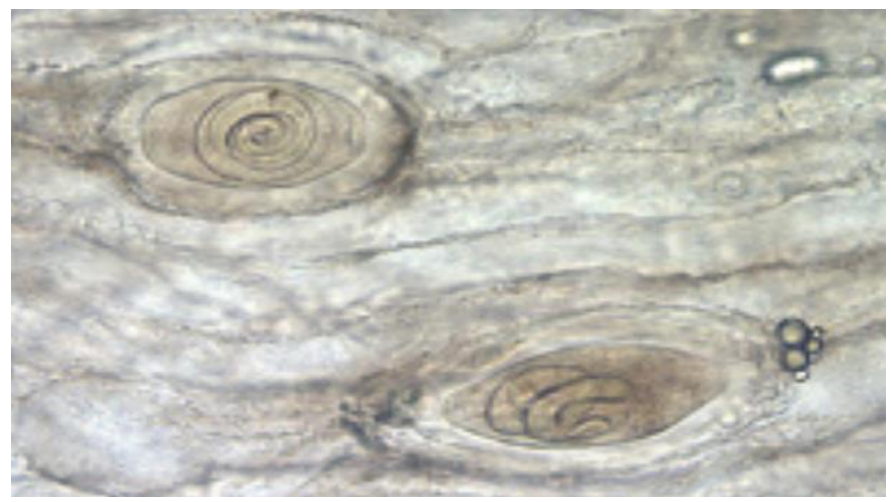

Fig. 3: Bear muscle showing the encysted larvae at 2oox magnification. United States Centers for Disease Control and Prevention (CDC). Available at: https://www.cdc.gov/dpdx/ trichinellosis. Public domain.

\section{Life style}

Adults and larvae parasitize themselves on the same host. When the host is infected, it becomes the terminal host first and then becomes the terminal host. The life cycle of Trichinella spiralis in humans and other host animals consists of three consecutive stages: the intestinal stage, the extraintestinal stage, and the encapsulation stage (Fig. 4). Life cycle of the parasite begins with the intestinal stage in the stomach and small intestine, where gastric acids and pepsin release the larvae encased in the striated muscles (Peters and Pasvol 2007). The released larvae invade the mucosa of the small intestine and grow into adults within one week and begin to mate and reproduce. The pregnant female releases the larvae into the lymphatic circulation and shifted to the striated muscles for subsequent development. After the larva enters the striated muscle fibres through the capillary, the release of vascular endothelial growth factor-stimulated by the cytokine causes the larva to form a cyst (Capo et al. 1998). The cysts begin to calcify after 6-9 months, but the larvae can remain viable for up to 11 years. The host is infected by eating cysts containing infectious larvae. The cysts are

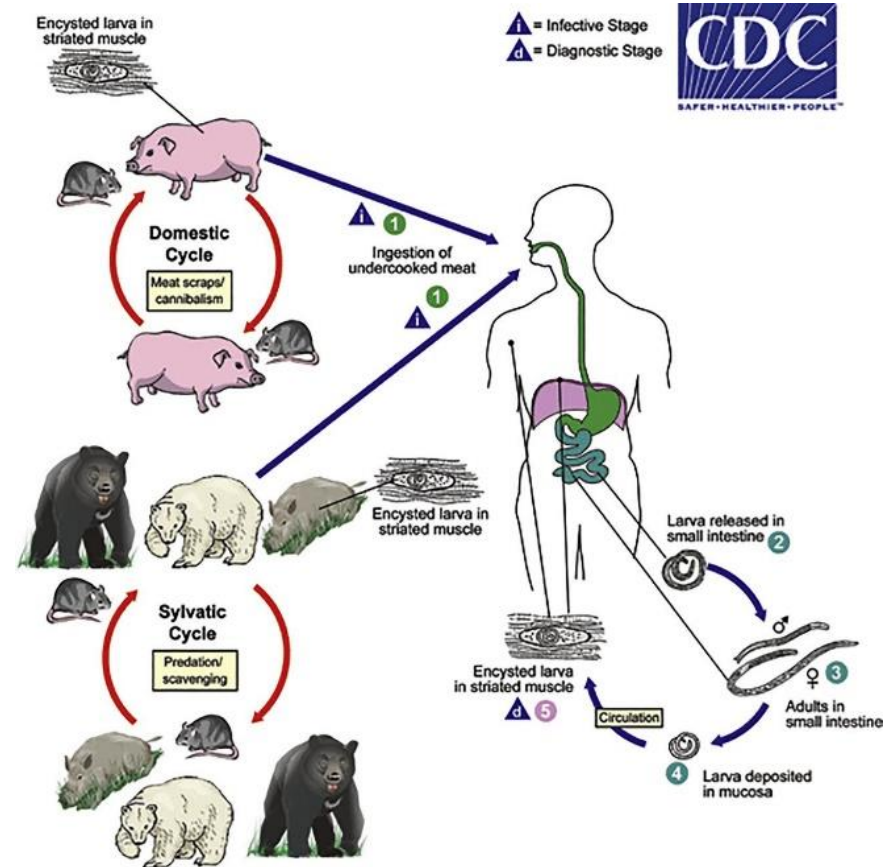

Fig. 4: The domestic and sylvatic life cycles of Trichinella species. United States Centers for Disease Control and Prevention (CDC). Available at: https://www.cdc.gov/dpdx/ trichinellosis. Public domain.

dissolved under the action of gastric acid and pepsin in the host, releasing the larvae into the intestines, where they can develop into adults in a short time.

\section{Epidemiology}

Trichinosis occurs worldwide, and it is estimated that about 10,000 cases occur every year. The cases are usually concentrated in people, who eat common animal infected meat. The host range and infection range of Trichinella spiralis are very wide. It is popular among various wild and domestic animals, and many marine animals worldwide. There are nine species of Trichinella disease. So far, 12 genotypes of Trichinella have been reported. The most common Trichinella spiralis that can cause human diseases is Trichinella swine. According to the Centers for Disease Control and Prevention, in the 1940s, about 400 cases of Trichinosis were reported every year, but the number of reported cases has dropped significantly since 2010. In the past 40 years, few Trichinosis cases have been reported, and the risk of the disease in commercially raised and properly prepared pork is very low. However, eating uncooked game, especially bear meat, can cause people to suffer from this disease. Table 2 shows the Trichinella species, their geographic distributions, and hosts.

\section{Pathogenesis}

The symptoms of animal infections are like those of humans. The severity of the pathogenic effects of Trichinella spiralis on the human body is related to factors such as the number of ingested larvae cysts and their vitality, as well as the immune function status of the host. 
Table 2: Trichinella species, their geographic distributions, and hosts. Data referenced from a previous report (Diaz et al. 2020).

\begin{tabular}{|c|c|c|}
\hline Species & $\begin{array}{l}\text { Geographic } \\
\text { distributions }\end{array}$ & Host \\
\hline Trichinella britovi & Europe, Asia, Africa & Wild and domestic swine, wild carnivores (bears, foxes, hyenas, lynxes, wolves) \\
\hline T. murrelli & North America & Black bears, grizzly bears, raccoons, red foxes, bobcats, coyotes, cougars, moose, wild boar \\
\hline T. nativa & $\begin{array}{l}\text { Arctic and subarctic } \\
\text { regions worldwide }\end{array}$ & $\begin{array}{l}\text { Arctic foxes, polar bears, black bears, brown bears, grizzly bears, walruses, sea ice- } \\
\text { associated seals, wolves, other wild carnivores }\end{array}$ \\
\hline T. nelsoni & Africa & Wild carnivores (wild dogs, hyenas, lions, tigers) \\
\hline T. papuae & $\begin{array}{l}\text { Papua New Guinea, } \\
\text { Taiwan, Thailand }\end{array}$ & Wild and domestic swine, saltwater crocodiles, soft-shelled turtles \\
\hline T. patagoniensis & Argentina & Cougars \\
\hline T. pseudospiralis & Worldwide & Birds, mammals, reptiles \\
\hline T. spiralis & Worldwide & Wild and domestic swine, horses \\
\hline T. zimbabwensis & Africa & Nile crocodiles, monitor lizards \\
\hline $\begin{array}{l}\text { Trichinella T8 } \\
\text { (unnamed genotype) }\end{array}$ & $\begin{array}{l}\text { Africa, South } \\
\text { Africa, Namibia }\end{array}$ & Lions, spotted hyenas (not detected in humans at present) \\
\hline $\begin{array}{l}\text { Trichinella T9 } \\
\text { (unnamed genotype) }\end{array}$ & Japan & Bears, wild carnivores \\
\hline
\end{tabular}

The larvae penetrate the intestinal wall and mature, causing extensive duodenal inflammation, mucosal congestion and oedema, bleeding, and even superficial ulcers. Vascular inflammation can occur where larvae migrate, causing a significant heterogeneous protein reaction (Shimoni and Froom 2015). The acute inflammation subsides with intramuscular cyst formation, and the systemic symptoms are eased, but the myalgia may last longer.

\section{Symptoms}

Initial symptoms of Trichinosis are gastrointestinal diseases, which usually occur 1-2 days after eating raw or undercooked meat from animals infected with Trichinella. These symptoms include nausea, diarrhoea, vomiting, and abdominal pain. The following typical Trichinosis symptoms usually occur within two weeks after eating contaminated meat and can last up to 8 weeks: muscle pain, fever, facial swelling, weakness or fatigue, headache, etc. Symptoms can range from very mild to severe and are related to the number of Trichinella eaten in the meat (Gottstein et al. 2009). Furthermore, many patients with Trichinosis do not show any symptoms.

\section{Diagnosis}

Diagnosis of animals before death is very difficult, and pig Trichinosis is often detected after slaughter. For this purpse, the diaphragm is examined with naked eyes, and when small white spots are seen between the muscle fibres, a microscope is used to observe whether there are cysts. In humans, acute Trichinosis usually occurs after eating raw or undercooked meat or having contact with the patient. Currently, commonly used laboratory testing method is ELISA. The positive coincidence rate of serum antibody detection with ELISA method can reach up to 9396\% (Dupouy-Camet et al. 2002).

\section{Fasciolopsis buski}

Fasciolopsis buski (Manning and Ratanarat 1970) is a member of the family Fasciolopsis and is parasitic in the small intestine of humans and pigs, most often in the duodenum. Occasionally, it can infect dogs and rabbits, where it is attached to the mucosa by powerful suckers or implanted in the mucosa, and is a large intestinal parasitic trematode (Malviya 1985). F. buski is known as a giant intestinal trematode (Lee et al. 2011) and is one of the largest digenean infecting humans globally, mainly confined to Asian nations, including China (Murugesh et al. 2007). The infection with F. buski develops through the consumption of raw or undercooked fishery foods contaminated with cysts containing the parasite (Chen et al. 2016). F. buski can also co-infect with other parasites, especially trematodes (Gupta et al. 1999). It can occur in the form of moderate and severe infections of the digestive tract, with the severe cases can adversely affect the immune system of the host, induce acute nephritis and even death, leading to major public health problems and losses in endemic areas (Karthikeyan et al. 2013).

\section{Morphology}

The adult worm body is flat, fat, and flesh-red when alive, grayish-white when fixed; body shape resembles ginger, so it is also called ginger worm (Fig. 5). The body of parasite is $20 \sim 75 \mathrm{~mm}$ long, 8 20 $\mathrm{mm}$ wide and $0.5 \sim 3.0$ $\mathrm{mm}$ thick, and it is the largest parasitic trematode in the human body. The oral sucker is located on the ventral surface of the front of the body, about $0.5 \mathrm{~mm}$ in diameter, and the ventral sucker is $2 \sim 3 \mathrm{~mm}$ in diameter, located near the back of the oral sucker, funnel-shaped, with well-developed muscles, and visible with the naked eye (Wiwanitkit et al. 2002).

\section{Life style}

Life style peculiarities of the worm are as follows: Immature eggs are released in the intestine and excreted via stool. After that, in water, eggs are embryonated, releasing miracidia that invade the appropriate intermediate host snail. In snails, parasites go through various stages of development (sporocysts, rediae and cercariae). The cercariae are discharged from snails and cysts and become metacercariae on aquatic plants. 


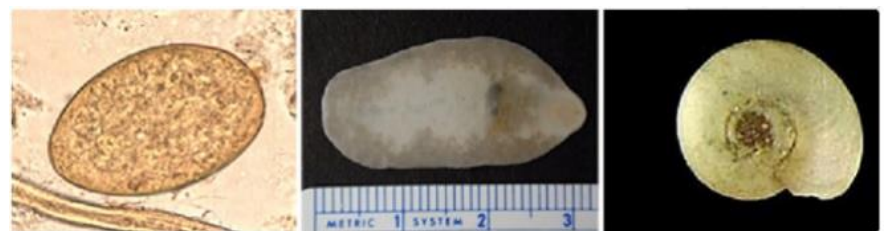

Fig. 5: Left: F. buski egg in an unstained wet mount. Center: Adult fluke of $F$. buski next to a scale. Right: Snail of the genus Hippeutis, an intermediate host for F. buski. (Credit: DPDx; Conchology, Inc., Mactan Island, Philippines).

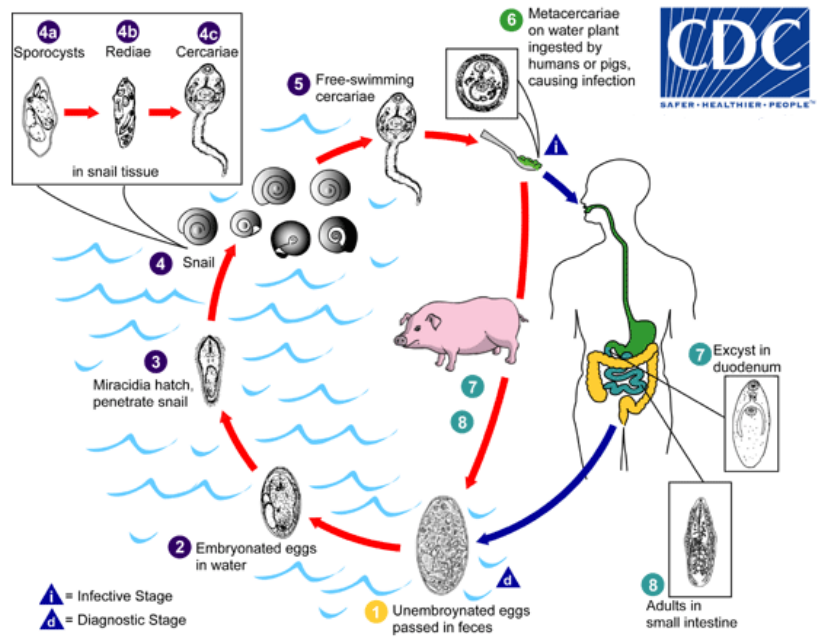

Fig. 6: Life cycle image and information of $F$. buski. United States Centers for Disease Control and Prevention (CDC). Available

https://www.cdc.gov/parasites/fasciolopsis/biology.html.

Mammalian hosts get infected by eating the metacercariae present on the aquatic plants. After ingestion, metacercariae enter the duodenum, leave the cyst and ascribe to the intestinal wall (Weng et al. 1989). They are evolved into adult flukes (20-75 $\mathrm{mm}$ x 8-20 $\mathrm{mm}$ ) over a period of about 3 months and adhere to the intestinal wall of mammalian hosts (humans and pigs). Adults have a lifespan of approximately one year (Fig. 6).

\section{Epidemiology}

The disease is endemic and mainly occurs in temperate and subtropical regions of Asian countries, such as Vietnam, Laos, Cambodia, Thailand, Myanmar, Malaysia, Bangladesh, India, Indonesia and the Philippines (Chai et al. 2009). In China, it is mainly distributed in the Yangtze River basin and southern China, such as Jiangsu, Zhejiang, Fujian, Anhui, Jiangxi, Yunnan, Shanghai, Hubei, Hunan, Guangxi, Guangdong, Guizhou, Sichuan, Chongqing, Hainan, and Taiwan. It also occurs in north of the Yangtze River in Shandong, Henan, Hebei, Shaanxi and Gansu (Yeh and Mitchell 2016).

\section{Host vectors}

The intermediate hosts, the flatworm snails, are widely distributed in ponds, marshes, ditches and paddy fields, often inhabiting under leaves of plants. Most of the aquatic plants can be used as the vector for the cysts of the ginger borer. Some aquatic plants commonly used for green fodder are essential vectors of swine infection (Achra et al. 2015).

\section{Pathogenesis}

Mechanical damage: The ginger sucker only sucks the intestinal mucosa with its strong oral and ventral suction discs, causing mechanical damage to the suction site, resulting in enteritis, intestinal mucosal detachment, bleeding and even abscess formation.

Mechanical blockage: When the intensity of infection is high, the intestine can be mechanically blocked, adversely affecting digestion and absorption and even causing intestinal rupture or intestinal entrapment and death.

Nutrient seizure: Due to its large size, the worm absorbs a vast amount of nutrients, which retards the growth of the sick animal and causes anaemia, wasting and malnutrition. The animal absorbs toxic effects of the worm, which can cause anaemia and oedema, inflammation, haemorrhage, ulceration and necrosis of the intestinal mucosa (Graczyk et al. 2000).

\section{Symptoms}

Ginger suckers mostly attack young pigs, resulting in stunted growth, sparse and lusterless coat, depressed spirit, low head, salivation, pale eye mucosa and dullness. Appetite of the affected animal is reduced, and digestion is poor, but sometimes animal feels hungery. There are signs of dysentery, thin faeces mixed with mucus. Affected animal shows signs of abdominal pain, diarrhoea, swelling and ascites ( $\mathrm{Wu}$ et al. 2020). The lactation yield and length of the affected sow is reduced, which affects the growth of piglets.

\section{Comprehensive preventive measures}

Manure disposal: In endemic areas, manure of diseased pigs is the primary source of transmission and should be composted and fermented as much as possible before being used as fertilizer (Chung et al. 1996). Also, human manure and pig manure should be equally avoided by mutual transmission between humans and animals. Regular deworming: In endemic areas, regular deworming should be carried out every year in spring and autumn (Manning and Ratanarat 1970). The intermediate hosts of flatworm should be eliminated. In the drier season of late autumn and early winter each year, digging pond mud to accumulate fertilizer and dry pond mud to kill snails is advised. In low-lying areas, where the pond water is not easily drained, snails can be exterminated by chemicals, such as copper sulfate with a concentration of $10 \sim 50 \times 10^{-5}$, $0.1 \%$ lime, $0.01 \%$ tea cakes and ammonia sulfate, lime nitrogen, etc (Roy et al. 2009).

\section{Cryptosporidiosis}

Cryptosporidiosis is caused by infection with protozoan parasite cryptosporidium in a wide range of host species, 
such as humans, livestock, companion animals, wildlife, birds, reptiles, and fish (Chalmers et al. 2013). In 1907, it was first described by Tyzzer in the small intestine of mice (Tyzzer 1912). The disease causes severe diarrhoea in mammals and distressing respiratory symptoms in birds, posing a serious threat to the health and production of the affected animals. It is also a common-established cause of sporadic gastroenteritis in immunocompromised humans, and symptoms include watery diarrhoea, nausea, abdominal pain, vomiting and low-grade fever (Lal et al. 2019).

\section{Morphology}

Cryptosporidium belongs to the Eucoccidiorida and Cryptosporidiidae in taxonomy. The Cryptosporidium species that can cause human infections are $C$. cuniculus, C. parvum, C. ubiquitum, C. viatorum, C. meleagridis, Cryptosporidium mink genotype, $C$. felis, $C$. hominis, $C$. canis, Chipmunk genotype I and $C$. muris. Several species related to ruminant infection include $C$. muris, $C$. andersoni, C. parvum, C. hominis, C. felis, and C. bovis.

Biological characteristics: Invasive forms of Cryptosporidium have an apical complex with polar rings, rhoptries, micronemes, conoid, and subpellicular microtubules. Locomotion in invasive forms occur by body flexion, gliding, or undulation. Male and female gametes develop independently. Homoxenous (one host life cycle) shows developmental stages just under the membrane of the host cell. Oocyst are without sporocysts and with four sporozoites, while microgametes are without flagella (Current and Garcia 1991). The oocysts of Cryptosporidium are rounded in shape and measure 4.2 $\mu-5.4 \mu \mathrm{m}$ in diameter (Fig. 7 ).

\section{Life style}

The oocysts of Cryptosporidium are transmitted among many hosts via the faecal-oral route. Furthermore, direct contact with faeces of infected animals or indirect transmission of contaminated food or water mediated by environmental contamination can also occur. Akin to coccidia, the developmental history of Cryptosporidium parvum includes excystation, merogony, gametogony, fertilization, oocyst formation, and sporogony. After ingesting infective Cryptosporidium oocysts, the environment of the gastrointestinal tract promotes oocysts to decyst and release four sporozoites. Sporozoites penetrate host cells and develop into trophozoites (uninucleate meronts), which form merozoites through asexual division. Invasive merozoites enter adjacent host cells to form type I (recycling) or type II meronts. Type II meronts enter host cells to form microgametes and macrogametes. Approximately $80 \%$ of the zygotes formed after fertilization of the microgametes, develop into thickwalled oocysts (environmentally resistant), released in faeces and transmit the infection from one host to another. Moreover, approximately $20 \%$ of the zygotes are transformed into thin-walled oocysts. These oocysts are released in the host and cause autoinfection (Current and Garcia 1991).

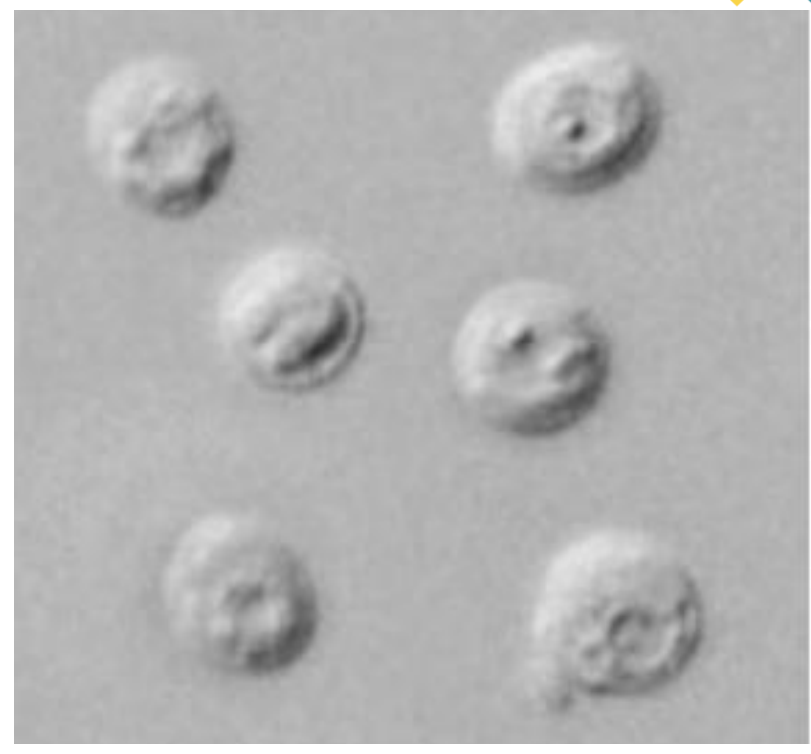

Fig. 7: Oocysts of Cryptosporidium parvum (Current and Garcia 1991).

\section{Epidemiology}

Cryptosporidium has a global distribution, and most countries have reported Cryptosporidium infections in humans and livestock. Cryptosporidiosis outbreaks have been recorded many times in the European Union countries, and their outbreaks are mainly associated with the spread of drinking or recreational waters, outdoor activities, animal contact, food consumption and personto-person spread (Rochelle and Giovanni 2014; Robertson and Chalmers 2013). Table 4 shows valid named species of Cryptosporidium.

Infection source: The infection source of Cryptosporidium is diseased animals or animals that discharge oocysts to the environment. These oocysts are highly resistant to the external environment.

Route of transmission: Oocysts are transmitted between infected animals and susceptible hosts orally through contaminated feed, drinking water or faeces and can even spread through the air.

Susceptible hosts: Cryptosporidium has a wide host range, which can parasitize more than 150 species of mammals, more than 30 species of birds, fish, and 57 species of reptiles. Additionally, Cryptosporidium does not have any apparent host specificity. For example, Cryptosporidium from quail can cause infection in chickens, ducks, mice, and other animals.

\section{Pathogenesis}

Cryptosporidium damages intestines of the host and induces a series of clinical symptoms, which may be caused by Cryptosporidium through altering the activity of host cells or absorbing nutrients from the gut.

Symptoms

Cryptosporidium infection usually causes watery diarrhoea, and the duration of symptoms is prolonged in 
Table 3: Related factors of Fascioliasis outbreaks described in the previous review (Siles-Lucas et al. 2021).

\begin{tabular}{llll}
\hline Country & Year & Infected number (species) & Attributed source \\
\hline HUMAN OUTBREAKS & & & \\
China & 2011 & 29 & Wild vegetables \\
Turkey & 2011 & 24 & Wild watercress \\
Romania & 2010 & 4 & NS \\
ANIMAL OUTBREAKS & & & \\
Brazil & 2016 & 19 & Water \\
Italy & 2014 & NS (sheep) & Pasture \\
Croatia & 2007 & 20 (sheep) & NS \\
\hline
\end{tabular}

NS, not stated.

Table 4: Valid named species of Cryptosporidium (Sunnotel et al. 2006).

\begin{tabular}{llll}
\hline Cryptosporidium species & Size $(\mu \mathrm{m})$ & Host & Location \\
\hline C. andersoni & $5.5 \times 7.4$ & Bovines & Abomasum \\
C. baileyi & $4.6 \times 6.2$ & Birds & Cloaca, bursa,respiratory tract \\
C. canis & $5.0 \times 4.7$ & Canids, human & Small intestine \\
C. felis & $4.5 \times 5.0$ & Felids, human & Small intestine \\
C. galli & $8.0-8.5 \times 6.2-6.4$ & Birds & Proventriculus \\
C. hominis & $4.5 \times 5.5$ & Human & Small intestine \\
C. meleagridis & $4.5-5.0 \times 4.6-5.2$ & Birds, human & Intestine \\
C. molnari & $4.7 \times 4.5$ & Fish & Stomach \\
C. muris & $5.6 \times 7.4$ & Rodents, human & Stomach \\
C. parvum & $4.5 \times 5.5$ & Ruminants, human & Intestine \\
C. saurophilum & $4.2-5.2 \times 4.4-5.6$ & Lizards, snake & Intestinal and cloacal mucosa \\
C. serpentis & $4.8-5.6 \times 5.6-6.6$ & Snakes, lizards & Stomach \\
C. suis & $5.1 \times 4.4$ & Pigs, human & Small intestine \\
C. wrairi & $4.0-5.0 \times 4.8-5.6$ & Guinea pigs & Small intestine \\
C. bovis & $4.2-4.8 \times 4.8-5.4$ & Ruminants & Small intestine \\
C. scophithalmi & $3.0-4.7 \times 3.7-5.0$ & Fish & Intestine
\end{tabular}

people with weakened immunity. Other common symptoms include nausea, low grade fever, abdominal pain, and vomiting. Occasionally, symptoms of weakness, myalgia, headache, malaise and anorexia can be seen.

Cryptosporidium commonly infects young animals, such as calves and lambs. In these animals, symptoms include diarrhoea with lethargy, poor body condition, inappetence, fever and dehydration.

\section{Lesions}

In Cryptosporidium infection, the intestinal villi shrank and become merged. The epithelial lining of the intestinal mucosa turns into low columnar or cuboidal cells, degenerates or falls off. Moreover, the cecum, colon and duodenum can also be infected. These lesions cause the host to reduce the absorption of vitamin A and carbohydrates.

\section{Diagnosis}

Many infective oocysts excreted with faeces, and the low infectious dose indicates that Cryptosporidiosis is highly contagious. However, in immunocompetent animals, this disease is self-limiting. Therefore, the exact diagnosis can only rely on laboratory methods to observe the various stages of Cryptosporidium or use immunological techniques to detect antigens or antibodies.

Diagnosis before death: Saturated sucrose solution is used to collect Cryptosporidium oocysts in the faecal samples; these samples are examined under microscope. It is essential to recognize that oocysts are too small; therefore, an oil lens with 100ox magnification is recommended.
Postmortem diagnosis: Giemsa dye solution is used to stain the digestive tract mucosa smears scraped off. In positive samples, the cytoplasm of the parasites can be observed to be blue, containing several dense red particles. Immunologic and molecular biologic techniques: Specific tests, including acid-fast or fluorescent stains to stain faecal smears, Enzyme-linked immunosorbent assay (ELISA), Polymerase chain reaction (PCR), and immunochromatographic lateral flow assays are recommended to confirm diagnosis (Chalmers and Katzer 2013).

\section{Clonorchiasis}

Clonorchis sinensis, also known as the Chinese liver fluke, belongs to the family Opisthorchiidae. This parasite causesa series of hepatobiliary diseases in the hepatobiliary duct of the host. Clonorchiasis is a freshwater fish-derived zoonotic parasitic disease, and the parasite is one of the most important foodborne parasites, endemic in Eastern Asian countries, including China, Korea, Vietnam, Thailand and the Far East regions of Russia (Lun et al. 2005; Traub et al. 2009; Hong and Fang 2012; Qian et al. 2012). Based on the national survey in China in 2003, it is estimated that over 12 million people are infected with $C$. sinensis, and the prevalence of this parasite is expanding in this country.

\section{Morphology}

The adult $C$. sinensis is a leaf-shaped hermaphroditic trematode, $10-25 \mathrm{~mm}$ in length and $3-5 \mathrm{~mm}$ in width with 
an anterior oral sucker, a centrally located ventral sucker and genital pore.

The egg of $C$. sinensis is small, with an average of 29 microns $\times 17$ microns, shaped like a light bulb, brown, with an operculum cover at the upper end and a small protuberance at the back end, which contains miracidium.

\section{Life style}

With a relatively wide host range, $C$. sinensis can infect to several snail species and more than 100 freshwater fish species (intermediate hosts). Many of such fish species belong to the Cyprinidae family, such as minnows and carps. In addition to humans, $C$. sinensis can affect canines, felines, mustelids, pigs, piscivorous mammals as definitive hosts. The life cycle of the $C$. sinensis involves three types of hosts: freshwater snails, freshwater fish, and piscivorous mammals, including humans.

Many freshwater snails (belonging to order Mesogastropoda) can be used as the primary intermediate host. Miracidium enters the sporocyst stage, during which it reproduces asexually. The asexual reproduction only occurs during summer, when the eggs develop to mature cercariae and are released into the water (Liang et al. 2009). Once the cercariae encounter the second intermediate host, they penetrate the skin mucosa of the fish, encyst as metacercariae and develop into mature metacercariae. At this time, when mammals ingest infected fish or fish meat, the metacercariae excyst in the duodenum and rapidly migrate into the intrahepatic bile ducts within 10-20 mins, develop into adults and begin to spawn. It is reported that $C$. sinensis lays about 4000, 2400 and 1600 eggs a day in humans, cats and guinea pigs, respectively (Kim et al,2011). The eggs flow to the intestines with bile and discharge water through faeces to continue the life cycle. Adults may survive in humans for over 26 years (Attwood and Chou 1978), as shown in Fig. 8.

\section{Epidemiology}

Clonorchis sinensis is a widespread parasite of humans, dogs, and cats in the southeast of Asia. It is extraordinarily common in China and is also found in Korea and Japan.

In the survey conducted in Guangdong, China, from 2004 to $2012,15,435$ out of 179756 people were infected, with an infection rate of $8.6 \%$. In the survey of Guangxi from 2005 to $2014,14,234$ of 144,073 people were infected, with an infection rate of $9.9 \%$. In addition, the population infection rates in Hunan, Jilin, Heilongjiang, Liaoning and Zhejiang were 34.3 (3195/9313), 18.5 (1979/10694), 12.4 (518/4177), 3.1 (123/3972) and 2.3\% (19/821), respectively (Tang et al, 2016). It is speculated that the high infection rate in endemic areas is related to local eating habits, including eating raw or half-raw freshwater fish and shrimp, which may also be an important reason for the widespread prevalence of the disease.

The epidemic level of Clonorchiasis in China is on the rise. Two national surveys show that the population's

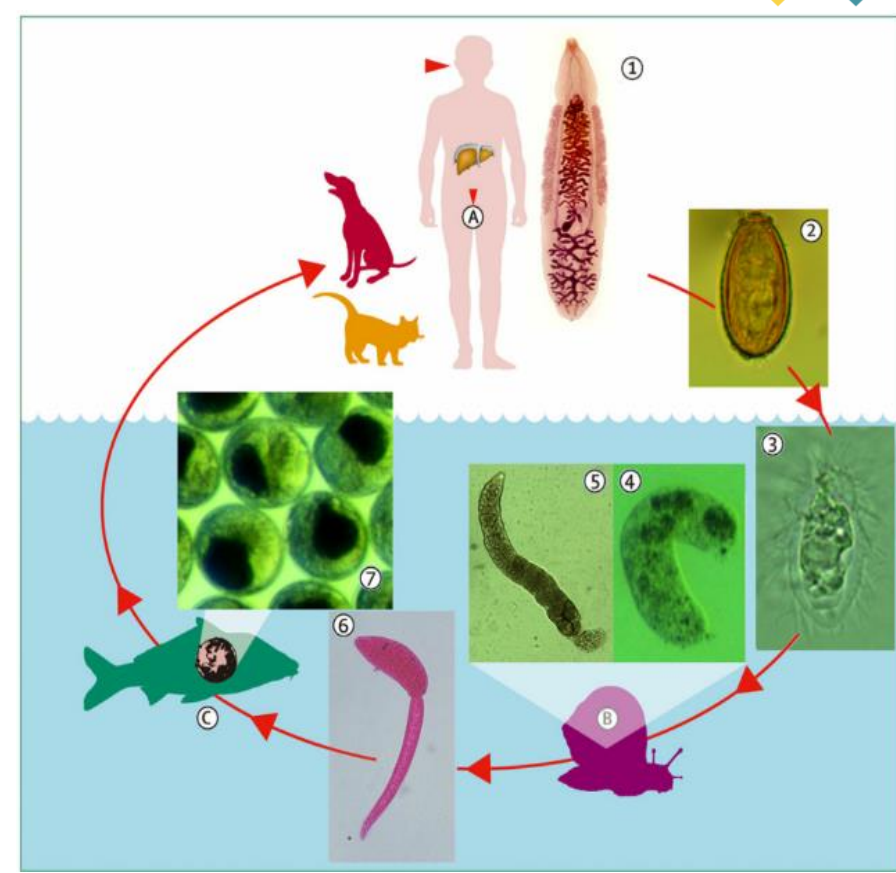

Fig. 8: Life cycle of Clonorchis sinensis. (1) lives mainly in the intrahepatic bile duct and gall bladder. Fully mature egg with miracidium (2) excreted in the fecal environment (water) and enters the body of the (B) first intermediate host, the freshwater snail. The miracidium (3) is discharged from the egg and enters snail's gastrointestinal tract, where it develops into sporocysts (4) and then into rediae (5). A large number of motile cercariae (6) are released into the water. Free-swimming cercariae invade the skin, fins, or muscles of freshwater fish as intermediate host (C) and develop into metacercariae (7). Consumption of raw or undercooked metacercariae infected freshwater fish can transmit the infection in the definitive host. Modified from Lun et al. (2005).

infection level is rising significantly, from $0.31 \%$ in the first survey to $0.58 \%$ in the second survey. Guangdong, in particular, showed the rise from $1.82 \%$ to $5.35 \%$ (Chen et al. 2012). It shows that the prevention and treatment of the disease have not been paid enough attention. Moreover, the level of prevention and control of Clonorchiasis in China lags behind other major foodborne Clonorchiasis endemic countries. For example, the main foodborne parasitic disease in South Korea is Clonorchiasis sinensis, but its epidemic level has dropped from $4.6 \%$ in 1971 to $2.4 \%$ in 2004 (Qian et al. 2014). In Thailand, the main endemic country of Retroclonorchiasis in muskcats, it fell from $14 \%$ in the early 198 os to $8.7 \%$ in 2009 (Sithithaworn et al. 2012). The situation of prevention and control of Clonorchiasis in China is grim, which should be paid more attention (Chen et al. 2012).

\section{Pathogenesis}

After infecting the host, Clonorchis sinensis moves in the bile duct through the suckers of the head and abdomen, causing mechanical damage to the bile duct epithelium. When the larvae mature, the injury is more serious, which can cause bile duct epithelial ulcers (Liu et al. 2010). With the advancement in the course of the disease, many adults and eggs block the bile duct, resulting in increased bile 
duct pressure. Consequently, eggs enter the surrounding liver tissue through ulcers, inducing numerous inflammatory cell infiltration to cause granuloma reaction; the bile duct obstruction will also cause bacterial infection, further aggravating bile duct injury. Repeated mechanical injury and inflammatory stimulations are important factors leading to bile duct epithelial hyperplasia and cholangiocarcinoma (Pan et al. 2017; Liu and Sun 2016). Clonorchis sinensis moves in the bile duct and continuously produces excretory/secretory products (ESPs). The researchers isolated 39 known functional proteins from ESPs, including glucose metabolic enzymes, detoxifying enzymes, structural proteins, etc., which play an important role in the interaction between host and parasites (Tang et al. 2016).

\section{Symptoms}

Symptoms of Clonorchiasis are associated with worm load. Most infections are mild and asymptomatic. The clinical manifestations are associated with inflammation, periodic obstruction of the bile ducts, mechanical damage caused by the mucosal tissue nutrition by worms, toxic effects of the worm's metabolic products, or byproducts, and secondary bacterial infections. In mild cases, symptoms include mild abdominal manifestations. Patients with a high burden of worms often present with other nonspecific symptoms such as abdominal pain (especially in the upper right abdomen) and various physical symptoms (such as headache, dizziness, etc.). Prolonged infection can exacerbate symptoms and cause hepatomegaly and malnutrition. It can sometimes increase the incidence of biliary complications (e.g. cholelithiasis, cholangitis, cholangiocarcinoma, and cholecystitis etc.), liver abscesses, and pancreatitis.

\section{Diagnosis}

The etiological diagnosis of Clonorchiasis sinensis is mainly based on the detection of eggs in faeces. The eggs in faeces are collected by precipitation method, floating method, washing method, aldehyde ether concentration method, modified Kato method, and the morphological characteristics of eggs observed under a microscope. This method is also the standard method for detecting Clonorchiasis sinensis. The immunological diagnostic methods of Clonorchiasis include intradermal test, indirect hemagglutination (IHA) test, ELISA, immunofluorescent antibody technique, (immunofluorescence technique), immune colloidal gold technique and serum circulating antigen detection. The widely used methods are ELISA and immuno-colloidal gold technique. ELISA mainly includes dot-ELISA, biotinavidin-ELISA, monoclonal antibody-ELISA, FAST-ELISA and cysteine protease-ELISA. Immunogold techniques include immunocolloidal gold chromatography (immunochromatography), dot immunogold silver staining (Dot-IGSS) and dot immunogold filtration assay (DIGFA).

\section{Cysticercus}

The causative agent of Cysticercosis cellulosae is the larva of Taenia solium, which is parasitic in the human body (Héctor et al. 2003). Pigs and wild boars are the main intermediate hosts, followed by dogs, camels, cats, and humans (Assana et al. 2019). Humans are the final host of Taenia solium. These organisms parasitize the striated muscles, heart, brain, eyes and other human and pig organs (Clinton et al. 2017). It not only affects the pig industry but also poses a huge threat to the human health.

\section{Morphology}

Cysticercus cellulosae is commonly known as cysticercosis. Mature Cysticercus cellulosae has an oval shape and is of a soybean size $(6-10 \mathrm{~mm} \times 6 \mathrm{~mm})$. It is characterized by a translucent cyst filled with liquid. The wall of the cyst is a thin film. There is a milky white nodule of the size of a round millet grain on the wall. There are 4 circular suction cups on the head section in the turned head section, and there are many small endoplasmic hooks on the top protrusion at the front end, arranged in two circles. The adult Cysticercus measures 2$5 \mathrm{~m}$ in length and some can grow up to $8 \mathrm{~m}$. There is 70o1000 nodal plate in the bug. The scolex is a spherical shape with a diameter of $1 \mathrm{~mm}$, and there are 20-25 horny little hooks on the rostellum. There are 4 sucking discs in the rear of the rostellum. The eggs are round or slightly elliptical with a diameter of $35 \mu \mathrm{m}$.

\section{Life cycle}

The adult parasitizes in the anterior part of small intestine and its cephalic segment is buried deep in the mucosa. Worm eggs or gravid proglottid are discharged with faeces and contaminate the ground and food. The intermediate host (mainly pig) swallows the eggs, or gravid proglottid can enter the intestinal wall under the action of the secretions of oncosphere. It enters the lymphatic and blood vessels and is carried to the muscles, heart, brain and throughout the body with blood circulation. After 2 months, it develops into infectious mature Cysticercus.

\section{Epidemiology}

Cysticercus disease is mainly prevalent in 18 countries and regions, where pork is the main food. This disease has been recorded in most provinces and autonomous regions of China, and it is more severe in the north. According to statistics, the national economic loss caused by cysticercosis can reach more than 80 million yuan every year. The epidemic of this disease has the following characteristics:

Pigs can be infected by ingestion of eggs in feed contaminated with human faeces. Human may become infected with Cysticercus by eating raw or under-cooked infected pork. The infection of this disease can occur in all 
Table 5: The prevalence data of Cysticercosis in some Asian countries (Rajshekhar et al. 2003).

\begin{tabular}{lccc} 
Country & Human Cysticercosis (\%) & Taeniasis (\%) & Porcine Cysticercosis (\%) \\
\hline China & $3-/ 4$ & $0.112(0.06-/ 19)$ & $5.4(0.8-/ 40)$ \\
Indonesia & $1.7-/ 13$ & $0.8-/ 23$ & $0.02-/ 2.63$ \\
Vietnam & $5-/ 7$ & $0.5-/ 6$ & $0.04-/ 0.9$ \\
India & NA & 2 & 9.3 \\
Nepal & NA & $10-/ 50$ & 32.5 \\
Korea & 3 & NA & NA \\
\hline
\end{tabular}

NA, not available

Table 6: Types of Echinococcosis currently prevalent and their approximate geographic range (Romig 2003).

\begin{tabular}{lll}
\hline Causative agent & Term for disease & Geographical range \\
\hline Echinococcus granulosus (species group) & Cystic echinococcosis (hydatidosis, hydatid disease) & Cosmopolitan \\
Echinococcus multilocularis & Alveolar echinococcosis & Northern hemisphere \\
Echinococcus vogeli & Polycystic echinococcosis & Central and South America \\
Echinococcus oligarthrus & Polycystic echinococcosis & Central and South America \\
\hline
\end{tabular}

seasons, but it tends to increase in the warm season when the environment is suitable for survival and development of eggs. The disease is generally sporadic, but endemic in some places, and its severity is positively correlated with the number of local tapeworm patients. Pigs are susceptible animals under natural conditions, and Cysticercus can survive in pigs for 3-5 years. Wild boars, dogs, and cats can also be infected. Although humans can serve as intermediate hosts, the disease is often fatal. Table 5 shows the prevalence data of Cysticercosis in some Asian countries.

\section{Pathogenesis}

Pathogenesis of Cysticercus disease varies remarkably with the number and location of Cysticercus parasitism. The migration of hyacercaria in vivo at the early stage of the disease can cause tissue damage. The pathogenicity of mature Cysticercus often depends on the location of the parasite, followed by their number. When parasite is in the brain, it can cause neurological symptoms and reduce the defence capability of the body by damaging integrity of the brain.

Moreover, it can cause death if brain lesions become severe. When it is parasitic in the eyes, it can cause visual disturbance and even blindness. When parasitic in the muscles and subcutaneous tissue, there are generally no obvious pathogenic effects.

\section{Clinical symptoms}

Generally, no obvious clinical symptoms are seen in pigs in the early stage of the disease. However, pigs infected with Cysticercus can show obvious clinical signs such as malnutrition, anemia, slow growth, and edema in the later period. Moreover, Cysticercus can parasitize in the lungs and throat, and can cause dyspnea, hoarseness and dysphagia. Cysticercus parasitization in the tongue can cause feeding difficulties. Cysticercus parasitized in the myocardium can cause blood circulation disorders. Cysticercus parasitized in the brain can cause epilepsy, acute encephalitis and even death. If Cysticercus is parasitized in the small intestine of humans, it can causenutrients depriviation and the secreted toxins can result in weight loss, abdominal pain, indigestion, and diarrhea. When Cysticercus parasitizes in the human brain, it can cause headache, mobility impairment, paralysis and other neurological symptoms.

\section{Diagnosis}

Blood and cerebrospinal fluid examination: Routine blood examination showed normal haematological values of most patients, but eosinophil counts were slightly elevated in a few patients. Moreover, cerebrospinal fluid pressure, lymphocyte and protein contents in patients with cerebral Cysticercosis were significantly increased, while sugar and chloride levels were within normal range.

Etiological examination: Fecal examination is carried out to check for eggs or nodules in the stool. Patients with subcutaneous and muscle Cysticercosis can undergo a subcutaneous nodule biopsy.

Immunologic tests: The purified Cysticercus fluid or serum can be used as an antigen to perform the intradermal test (ID), indirect hemagglutination test (IHA), enzyme-linked immunosorbent test (ELISA), enzyme immunoassay (EIA) to detect specific antibodies of Cysticercus in serum and cerebrospinal fluid of patients. Imageological examination: This examination includes CT scan and MRI of the skull, ophthalmoscopy, slit lamp, or B-mode ultrasonography.

Pathological examination: Subcutaneous nodules can be examined through biopsy, and Cysticercus cephalon in the lumen can be confirmed by pathological section.

\section{Schistosomosis Japonicum}

Schistosomiasis is a parasitic disease that seriously endangers the health of humans and livestock (Ayé et al. 2020). It has a high incidence in the Middle East, South America, and Southeast Asia, especially sub-Saharan Africa. Schistosomiasis is endemic in 76 countries around the world and approximately two hundred million people were infected. Infectious larvae grow in freshwater snails and then penetrate the skin of the final host. Adults colonize the mesentery or pelvic vein, where females lay eggs, which are secreted in feces or urine. Eggs trapped in surrounding tissues and organs, such as the liver and 
bladder, can cause an inflammatory immune response, leading to diseases of the intestine, liver and spleen or genitourinary system (Mcmanus et al. 2018).

\section{Morphology}

Adult organisms are dioecious and long cylindrical. The male is stubby, milky white, with a smooth body surface, $10-20 \mathrm{~mm}$ long and $0.5-0.55 \mathrm{~mm}$ wide. The female is slender and darker than the male, with a small front end and a thick rear end.

\section{Life style}

Oncomelania snails are the only intermediate host of Schistosoma japonicum. Humans or mammals are parasitized by adults and are called terminal hosts. Oncomelania snails are parasitized by vegetative larvae, and are called intermediate hosts. These two methods of reproduction alternate with each other, called generational alternation (Mcmanus et al. 2018). The approximate course of its life history is that patients or livestock infected with Schistosomiasis excrete worm eggs with their faeces or urine, the excrement contaminates the water, the worm eggs are taken into the water, and when they meet the right temperature, they will decompose, releasing the mircaria in the water (Fig. 9).

The miracidia can penetrate the snails, continue to develop into mother spores, and then reproduce asexually to produce daughter spores and cercariae. Cercariae can escape from the spiral body and swim freely in the water under suitable temperature, humidity, light, and other external environmental conditions. The water body containing cercariae is called diseased water. If people or other susceptible animals come in contact with the diseased water, cercariae penetrate the skin and turn into young worms after entering the skin. After migration and a period of growth and development, they finally settle in the portal vein system or the bladder, pelvic venous plexus, and develop into adult worms. The male and female adults mate together. Eggs and worms can be excreted with feces or deposited in the liver to form nodules (McCreesh et al. 2015). In general, the life history of Schistosomiasis includes six stages: adult, egg, miracidia, larvae, cercariae, and juvenile worms. Among these, from egg to juvenile is asexual reproduction; mirabilia and cercariae can live freely in the natural water for a short time.

\section{Epidemiology}

Schistosomiasis is considered a neglected tropical disease that occurs mainly in tropical and subtropical regions (Fig. 10). About 779 million people worldwide are at risk of infection, and more than 250 million people are infected with Schistosomiasis. Schistosomiasis japonicum was endemic in Japan until it was eliminated in the late 1970s with effective control measures (Rollinson et al. 2013). The remaining species have a low global prevalence and are currently distributed mainly in South Africa, West and Central Africa and the Mekong River in southern

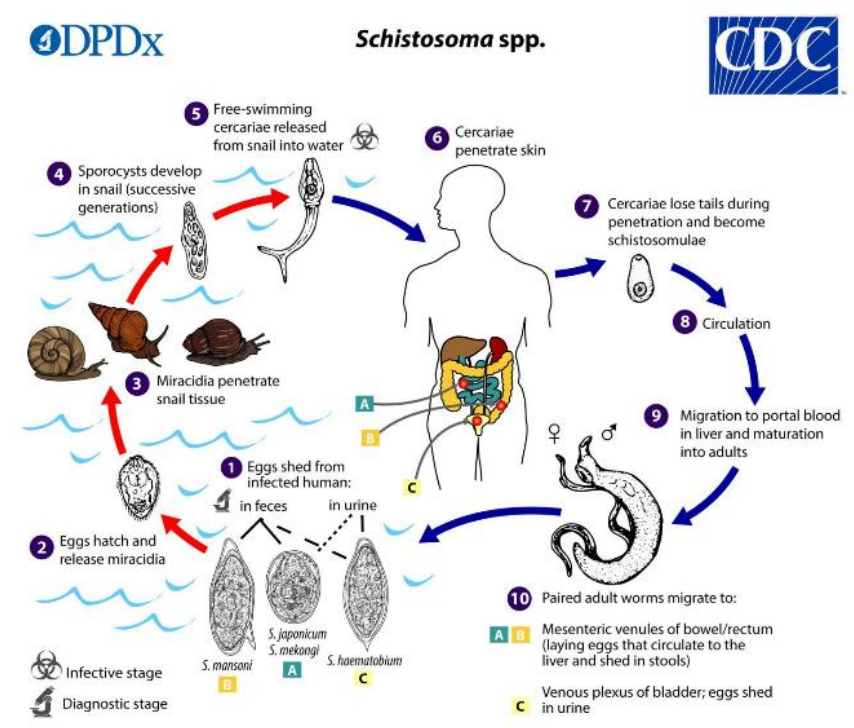

Fig. 9: The lifestyle of Schistosomosis japonicum (CDC 2018)

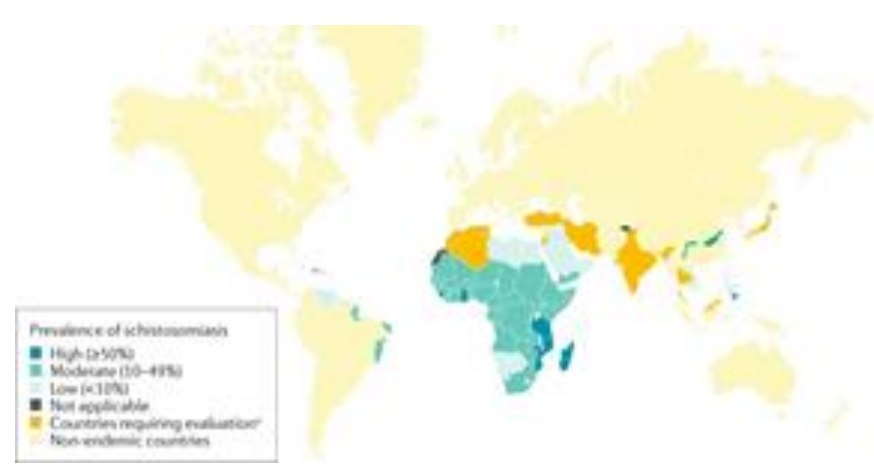

Fig. 10: Global distribution of Schistosomiasis in 2012 (Mcmanus et al. 2018)

Cambodia and the Lao People's Democratic Republic. The main intermediate hosts appear to be dogs and domestic pigs, but other animals, particularly cattle, may also be involved in transmission of the infection (Latif et al. 2013).

\section{Pathogenic mechanism}

After the cercariae penetrate the intact skin, part of the infected larvae will die in the skin, and the rest will directly enter the venous circulation through small blood vessels or lymphatic vessels. The larvae flow through the blood to the mature part of the liver. In the skin, the innate immune response to dying or dead larvae can cause an allergic reaction, and when exposed to water containing cercariae, a popular itching reaction on the body part, called cercariae dermatitis, is noted (Mcmanus et al. 2018). Symptomatic acute Schistosomiasis is also known as Katayama fever or Katayama syndrome. It usually occurs when a person comes in contact with a Schistosoma species for the first time, between 2 weeks and 3 months after exposure. These symptoms are caused by a systemic allergic reaction and the formation of immune complexes, which are responses to antigens released when the young Schistosomiasis worms migrate or deposit their eggs. Symptoms are usually accompanied by eosinophilia and transient lung infiltration (Chen et al. 2021). 
Symptoms

Many people show no symptoms when they are first infected. However, within a few days after infection, they may develop a rash or itchy skin. Symptoms that may occur within 1-2 months after infection include fever, chills, cough, and muscle pain. If left untreated, Schistosomiasis can last for many years. Symptoms and signs of chronic Schistosomiasis include abdominal pain, enlarged liver, blood in the stool or urine, and urination problems. Chronic infection can also lead to an increased risk of liver fibrosis or bladder cancer.

\section{Diagnosis}

There are two main diagnostic methods for Schistosoma japonicum in animals based on serology and parasitology. The parasitology-based diagnostic tool is the gold standard, but it takes a long time, and the false-negative result rate is high at low infection intensity, due to which it is gradually replaced by serological diagnosis. Still, the serological diagnosis has its shortcomings like crossreaction of other parasitic diseases, false-positive results, and problem of distinguishing the current infection from the previous infection. Therefore, there is an urgent need for more accurate and sensitive diagnostic methods to control Schistosomiasis (Mcmanus et al. 2018).

\section{Parasitological diagnosis}

The diagnosis based on parasitology refers to examining Schistosome eggs in the faeces or tissues of terminal host to be tested and examining the eggs after slaughter. The detection methods of eggs in faeces include the direct smear method, sediment collection method and nylon sieve egg method. KKM is usually used to detect faecal eggs. Pathogen detection is the most accurate diagnostic method for Schistosomiasis. The detection of eggs or miracidia hatched from faeces of the final host is the gold standard for Schistosomiasis diagnosis (Chen et al. 2021).

\section{Serological diagnosis}

Serological diagnosis is the use of the immune response of the specific combination of antigens and antibodies to detect diseases. It is also an important means for diagnosing and detecting Schistosomiasis. To improve the diagnosis efficiency, a variety of serological methods for the diagnosis of Schistosomiases such as IHA, ELISA, colloidal gold test strips, and nucleic acid detection have been developed (Chen et al. 2021).

\section{IHA}

The IHA applies antigens (or antibodies) to the surface of red blood cells, converts them into sensitizing carriers, and then binds to the corresponding antibodies (or antigens). Then, the red blood cells gather together, and a visible agglutination reaction occurs. IHA is suitable for on-site diagnosis because it is low-cost, easy to operate, and does not require any expensive equipment (Chen et al. 2021).

\section{ELISA}

The basic principle of enzyme-linked immunosorbent assay is to specifically bind the analyte in the sample with the enzyme through the specific immune reaction between the antigen and the antibody. The colour reaction between the enzyme and the substrate is then used to determine the analyte content. Enzyme-linked immunosorbent assay has become a commonly used qualitative and quantitative analysis method. Many Schistosome antigens are used for diagnoses, such as soluble egg antigens, soluble worm antigen preparations and recombinant antigens. However, SEA may be more effective in terms of the sensitivity of the diagnosis of Schistosomiasis (Cai et al. 2019).

\section{Colloidal gold test strips}

Because of its simple operation, low cost, long-term storage potential, and unlimited experimental conditions, the test strip method is gradually becoming popular for on-site rapid screening and diagnosis of Schistosomiasis. Types of test paper methods include test paper dye immunoassay (DDIA), latex microsphere labeling method, and colloidal gold immunochromatography.

\section{Nucleic acid testing for diagnosis}

Nucleic acids are present in all developmental stages of Schistosomes. The life cycle of Schistosome and the peculiarity of its parasitic site determine Schistosome nucleic acid detection technology (Cai et al. 2019).

\section{Echinococcosis}

Echinococcus disease, also known as hydatid disease, is caused by the larval stage of Echinococcus granulosus, a tapeworm that is about $2-7 \mathrm{~mm}$ long. It is found in dogs (ultimate hosts) and sheep, cattle, goats and pigs, which are intermediate hosts (Grech-Angelini et al. 2019; Tamarozzi et al. 2020). Although most human infections are asymptomatic, chronic pancreatitis can cause harmful, slowly expanding cysts in the liver, lungs, and other organs that often remain unnoticed and ignored for many years (Matsumoto, 2020). The World Health Organization (WHO) has listed Echinococcosis as one of the 17 neglected diseases.

\section{Morphology}

The shape of hydatid cysts varies widely due to their parasitic parts. They are generally approximately spherical, with a diameter of $5-10 \mathrm{~cm}$. Cyst wall can be divided into two layers, the inner layer is the germinal layer or germ layer, and the outer layer is the milky white stratum corneum. Adult parasites are very small, with a total length of $2-6 \mathrm{~mm}$, consisting of a head and three to four segments. There are suckers, apical processes and small hooks on the top of the head. There are several apical glands on the apical processes, which are covered by a radial embryonic membrane (Fig. 11). 

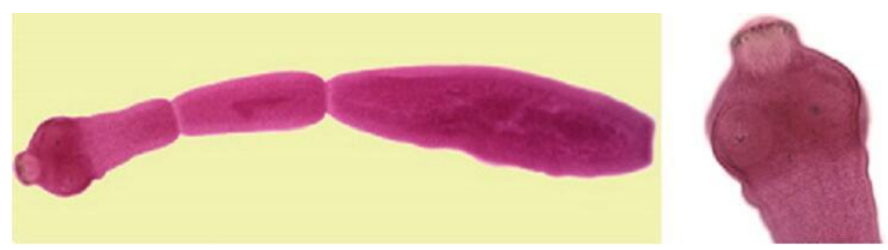

Fig. 11: From left to right are; an adult Echinococcus granulosus and the close-up of scolex section. United States Centers for Disease Control and Prevention (CDC). Available at: https://www.cdc.gov/parasites/echinococcosis/.

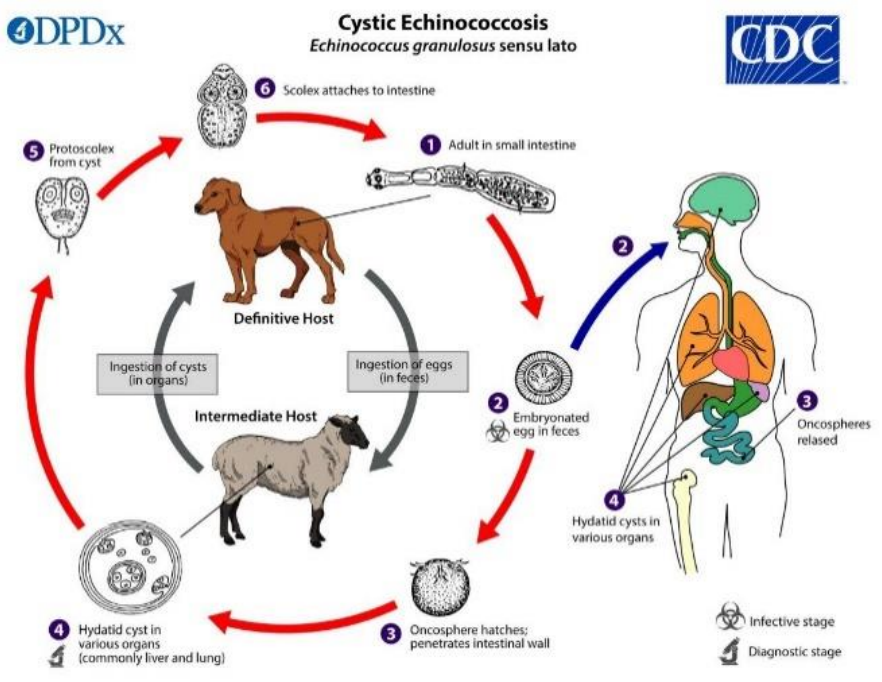

Fig. 12: Life cycles of Echinococcus spp (Hao et al. 2019).

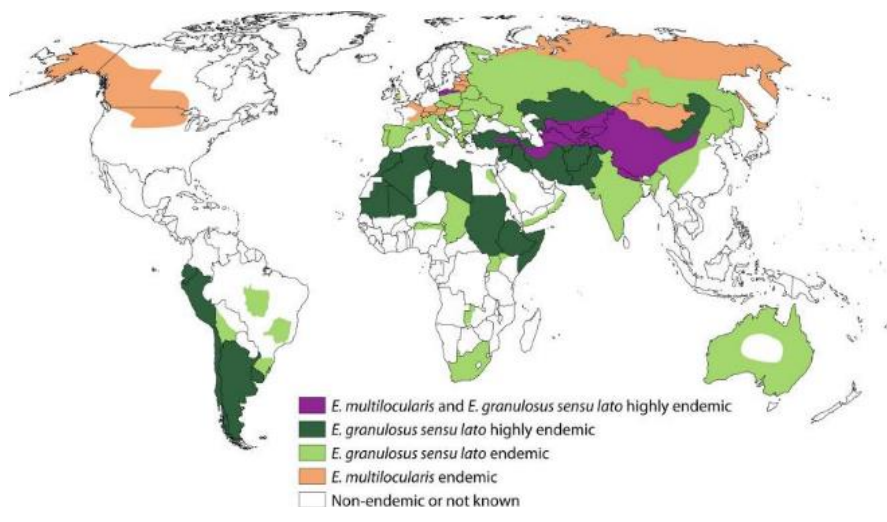

Fig. 13: Global distribution of Echinococcus granulosus sensu lato, responsible for Cystic Echinococcosis (CE), and Echinococcus multilocularis, responsible for Alveolar Echinococcosis (AE) (Woolsey and Miller 2020).

Life style

Carnivores (canines and cats) are the ultimate hosts of adult tapeworms, and herbivorous prey (ungulates, rodents and lagomorphs) are the intermediate hosts of tapeworms. However, in some unique and unusual circumstances, such as those reported in Turkana, Kenya, humans can act as intermediate hosts, but humans are usually not directly involved in transmission (Woolsey and Miller 2020). Adult worms develop in the intestine of their final host; the last segment (or anterior segment) of each worm matures to produce eggs, which are released into the external environment through faeces of carnivores. In turn, humans or intermediate hosts ingest eggs, which hatch in the intestines and release floats that pass through the portal vein and lymphatic vessels to reach the liver, where they usually settle and develop as larvae (metacysts or hydatid cysts). Occasionally, they may also reach the lungs, brain, bones or other organs of humans or intermediate hosts. The protoschi, the fertile form of the parasite, is asexually produced by the cysts and is released into the hydatid fluid; when it is finally ingested, the protoschi, with the help of bile salts, makes the scrotum everted. After the intestinal wall, they develop into mature egg-laying adults (Hao et al. 2019) (Fig. 12).

\section{Epidemiology}

Cystic Echinococcosis is distributed all over the world and is a major public health problem in some regions (Fig. 13). It is recorded in Peru, Chile, Argentina, Uruguay, southern Brazil, Mediterranean region, Central Asia, western China, and East Africa. It is an endemic problem. Cystic Echinococcosis has not been found in Antarctica. It has been eliminated through comprehensive control plans in Iceland, New Zealand, Tasmania, Falkland Islands and Cyprus; in addition, it has been previously recognized as endemic in France, Switzerland, Germany and Austria. The incidence of adverse events has doubled. As far as North America is concerned, the central and northern United States, the northwestern part of Alaska, and the northwestern part of Canada have long been endemic to E. multilocularis. It is expanding, at least in part due to increased and improved sampling efforts and targeting end hosts other than foxes (such as coyotes). Until recently, Cystic Echinococcosis was considered a mainstream human health problem in North America, except for Alaska, and Echinococcus has not been reported from Mexico or the southern United States.

\section{Pathogenic mechanism}

The pathogenic effects of Echinococcus on humans and animals include mechanical compression, toxic effects, and allergic reactions. The severity of symptoms depends on the size, location and number of hydatids. Echinococcus parasitizes mostly in the liver of animals, followed by the lungs. Mechanical compression can cause atrophy of the surrounding solid tissues and severe functional disturbances in the affected parts. After the metabolites are absorbed, the surrounding tissues will cause inflammation and systemic allergic reactions. The disease can be fatal, and the harm to humans is particularly obvious. Echinococcus multilocularis is more harmful to humans than Echinococcus granulosus.

\section{Symptoms}

Cystic Echinococcosis is usually asymptomatic, unless there are complications. Rupture of cysts can lead to infection or allergic reactions and fistula formation in adjacent structures (such as biliary tract, intestine, and 
bronchus). Mass effect on adjacent structures is the main mechanism for the usual symptoms of cysts. Incidental discovery of cysts during imaging studies conducted for other reasons are not uncommon.

\section{Diagnosis}

Imaging technology is essential for diagnosis of Echinococcosis. Ultrasound, which is relatively cheap and portable, is widely used to diagnose $\mathrm{CE}$ or $\mathrm{AE}$ induced liver lesions; X-rays are used for lung cysts. Both techniques are also used for diagnosis and population screening, and follow-up. Serology, which involves detection of specific antigens, is also used for Echinococcosis diagnosis (Mcmanus et al. 2003).

Magnetic resonance cholangiopancreatography (MCRP) is also useful in preoperative assessment of complications such as bladder-biliary fistula. This technique is also used in identifying bile duct obstruction. Like MCRP, endoscopic retrograde cholangio-pancreatography (ERCP) is also non-invasive and used for diagnostic purposes. Bladder-biliary fistulas become obvious only after the internal capsule ruptures (mainly $\mathrm{CE}_{2}$ and $\left.\mathrm{CE}_{3} \mathrm{~b}\right)$. The sensitivity and specificity of MRCP in detecting bladder-biliary fistulas at these stages are 75 and 95\%, respectively (Kahlfu et al. 2004).

Clinical laboratory analyss, including chemical and hematological tests, is non-specific in patients with Cystic Echinococcosis. For patients with biliary obstruction, increased levels of bilirubin, transaminase and gammaglutamyltransferase can be observed. In the case where the cyst leaks into the bile duct tree or the cyst ruptures, a significant increase in $\gamma$-glutamyltransferase, alkaline phosphatase and eosinophil counts can also be observed. However, such findings are usually not observed in cases of intact cysts.

\section{Conclusion}

Parasitic diseases mainly occur in areas dominated by animal husbandry, and the prevalence is usually higher in rural areas than that in urban areas. Attention needs to be paid to hygiene and deworming during the breeding process to avoid the widespread transmission of parasitic diseases among populations. In this chapter, description regarding the main zoonotic parasites that are prevalent in China, including Toxoplasmosis, Trichinosis, Fasciolopsis buski, Cryptosporidiosis, Clonorchiasis, Cysticercus, Schistosomosis japonicum and Echinococcosis has been given. It is hoped that more knowledge of parasites will be widely known to everyone, which will help reduce the incidence of parasitic diseases in livestock and humans worldwide.

\section{REFERENCES}

Achra A et al., 2015. Fasciolopsiasis: Endemic focus of a neglected parasitic disease in Bihar. Indian Journal of Medical Microbiology 33: 364-368.
Aguirre AA et al., 2019. The one health approach to Toxoplasmosis: Epidemiology, control and prevention strategies. Ecohealth 16: 378-390.

Arefkhah $\mathrm{N}$ et al., 2020. Seroprevalence and associated risk factors of Toxocariasis among nomads in BoyerAhmad County, southwest Iran. Transactions of the Royal Society of Tropical Medicine and Hygiene 114: 372-377.

Assana E et al., 2019. Prevalence of porcine Taenia solium and Taenia hydatigena Cysticercosis in Cameroon. Preventive Veterinary Medicine 169: 10469o.

Attwood HD and Chou ST, 1978. The longevity of Clonorchis sinensis. Pathology 10: 153-156.

Ayé P et al., 2020. Patients with severe Schistosomiasis mekongi morbidity demonstrating ongoing transmission in Southern Lao People's Democratic Republic. Acta Tropica 204: 105323.

Burger E et al., 2018. Loss of Paneth cell autophagy causes acute susceptibility to Toxoplasma gondii-mediated inflammation. Cell Host Microbe 23: 177-19o.

Cai $P$ et al., 2019. Mcmanus comparison of Kato Katz, antibody-based ELISA and droplet digital PCR diagnosis of Schistosomiasis japonica: Lessons learnt from a setting of low infection intensity. PLoS Neglected Tropical Diseases 13: eooo7228.

Capo VA et al., 1998. Trichinella spiralis: Vascular endothelial growth factor is up-regulated within the nurse cell during the early phase of its formation. Journal of Parasitology 84: 209-214.

Chai JY et al., 2009. Foodborne intestinal flukes in Southeast Asia. Korean Journal of Parasitology 47: 69.

Chalmers RM and Katzer F, 2013. Looking for Cryptosporidium: The application of advances in detection and diagnosis. Trends in Parasitology 29: 237-251.

Chen YD et al., 2012. Analysis of the results of two nationwide surveys on Clonorchis sinensis infection in China. Biomedical and Environmental Sciences 25: 163-166.

Chen MX et al., 2016. Identification and characterization of microRNAs in the zoonotic fluke Fasciolopsis buski. Parasitology Research 115: 2433-2438.

Chen $\mathrm{C}$ et al., 2021. Reviews and advances in diagnostic research on Schistosoma japonicum. Acta Tropica 213: 105743.

Chung PR et al., 1996. Segmentina (polypylis) hemisphaerula (Gastropoda: Planorbidae): A new molluscan intermediate host of a human intestinal fluke Neodiplostomum seoulensis (Trematoda: Diplostomatidae) in Korea. Journal of Parasitology 82: 336-338.

Clinton WA et al., 2017. Diagnosis and Treatment of Neurocysticercosis: 2017 Clinical Practice Guidelines by the Infectious Diseases Society of America (IDSA) and the American Society of Tropical Medicine and Hygiene (ASTMH). Clinical Infectious Diseases An Official Publication of the Infectious Diseases Society of America 2018: 8.

Current WL and Garcia LS, 1991. Cryptosporidiosis. Clinical Microbiology Reviews 4: 325-358. 
Diaz JH et al., 2020. The disease ecology, epidemiology, clinical manifestations, and management of Trichinellosis linked to consumption of wild animal meat. Wilderness and Environmental Medicine 31: 235-244.

Dimzas D et al., 2019. Human Trichinellosis caused by Trichinella britovi in Greece, and literature review. Journal of Helminthology 94: 1-4.

Dubey JP et al., 1998. Structures of Toxoplasma gondii tachyzoites, bradyzoites, and sporozoites and biology and development of tissue cysts. Clinical Microbiology Reviews 11: 267-299.

Dupouy-Camet J et al., 2002. Opinion on the diagnosis and treatment of human Trichinellosis. Expert Opinion on Pharmacotherapy 3: 1117-1130.

Ferguson DJ and Hutchison WM, 1987. The host-parasite relationship of Toxoplasma gondii in the brains of chronically infected mice. Virchows Archiv. A, Pathological Anatomy and Histopathology 411: 39-43.

Ferguson DJ, 2002. Toxoplasma gondii and sex: Essential or optional extra? Trends in Parasitology 18: 355-359.

Ferguson DJ et al., 2005. Maternal inheritance and stagespecific variation of the apicoplast in Toxoplasma gondii during development in the intermediate and definitive host. Eukaryotic Cell 4: 814-826.

Gottstein B et al., 2009. Epidemiology, diagnosis, treatment and control of Trichinellosis. Clinical Microbiology Reviews 22: 127e45.

Graczyk TK et al., 200o. Development of Fasciolopsis buski (Trematoda: Fasciolidae) in Hippeutis umbilicalis and Segmentina trochoideus (Gastropoda: Pulmonata). Parasitology Research 86: 324-326.

Grech-Angelini S et al., 2019. Identification and molecular characterization of Echinococcus canadensis G6/7 in dogs from Corsica, France. Parasitology Research 118: 1313-1319.

Gupta A et al., 1999. Fasciolopsis buski (giant intestinal fluke) - A case report. Indian Journal of Pathology and Microbiology 42: 359-360.

Hernández-de-Los-Ríos A et al., 2019. Toxoplasma Gondii: Influence of two major virulence factors (ROP16 and $\mathrm{ROP} 18)$ on the immune response of peripheral blood mononuclear cells to human Toxoplasmosis infection. Frontiers in Cellular and Infection Microbiology 9: 413.

Hong ST and Fang Y, 2012. Clonorchis sinensis and Clonorchiasis, an update. Parasitology International 61: 17-24.

Héctor $\mathrm{H}$ et al., 2003. Taenia solium cysticercosis. Lancet 362(9383):547-56

Kahlfu S et al., 2016. Diagnosis and treatment of Cardiac Echinococcosis. Heart 102: 1348.

Karthikeyan G et al., 2013. Intestinal infestation with Fasciolopsis buski leading to acute kidney injury. Journal of the Association of Physicians of India 61: 936.

Kim J et al., 2011. Correlation between discharged worms and fecal egg counts in human Clonorchiasis. PLoS Neglected Tropical Diseases 5: e1339.

Lal A et al., 2019. Local weather, flooding history and childhood diarrhoea caused by the parasite
Cryptosporidium spp.: A systematic review and metaanalysis. Science of The Total Environment 674: 300306.

Latif B et al., 2013. Autochthonous human Schistosomiasis, Malaysia. Emerging Infectious Diseases 19: 1340-1341.

Lee TH et al., 2011. Gastrointestinal: Fasciolopsis buski infestation diagnosed by upper gastrointestinal endoscopy. Journal of Gastroenterology and Hepatology 26: 1464 .

Liang $\mathrm{C}$ et al., 2009. Experimental establishment of life cycle of Clonorchis sinensis. Zhongguo Ji Sheng Chong Xue Yu Ji Sheng Chong Bing Za Zhi 27: 148-150.

Liu GX et al., 2010. Research progress on the relationship between three kinds of liver fluke infections and cholangiocarcinoma. Chinese Journal of Parasitology and Parasitic Diseases 28: 301-305.

Liu JX et al., 2016. Study of the development of Clonorchis Sinensis in susceptible animals and pathological changes in the liver. Journal of Pathogen Biology 11: 649-652.

Lun ZR et al., 2005. Clonorchiasis: a key foodborne zoonosis in China. Lancet Infectious Diseases 5: 31-41.

Matsumoto J, 2020 Intestinal Echinococcosis in a dog from Missouri. Journal of the American Veterinary Medical Association 256: 1041-1046.

Malviya HC 1985. The susceptibility of mammals to Fasciolopsis buski. Journal of Helminthology. 59: 1922.

Manning GS and Ratanarat C, 1970. Fasciolopsis buski (Lankester, 1857) in Thailand. American Journal of Tropical Medicine and Hygiene 19: 613-619.

Mcmanus DP et al., 2018. Schistosomiasis. Nature Reviews Disease Primers 4:13.

Mcmanus DP et al., 2003. Echinococcosis. Lancet 362: 1295-1304.

Mohammed OB et al., 2019. Seroprevalence of Toxoplasma gondii in household and stray cats of Riyadh, Saudi Arabia. Veterinaria Italiana 55: 241-245.

Montoya JG and Liesenfeld O, 2004. Toxoplasmosis. Lancet 363: 1965-1976.

Murugesh $\mathrm{M}$ et al., 2007. Endoscopic extraction of Fasciolopsis buski. Endoscopy http://10.1055/s-2006 $-945154$

Pan Y et al., 2017. Pathological observation of liver in mice infected with Clonorchis sinensis. Medical Innovation of China 14: 15-18.

Peters W and Pasvol V, 2007. Atlas of Tropical Medicine and Parasitology. Elsevier Mosby.

Pinto-Ferreira F et al., 2019. Patterns of transmission and sources of infection in outbreaks of human Toxoplasmosis. Emerging Infectious Diseases 25: 21772182.

Qian MB et al., 2012. The global epidemiology of Clonorchiasis and its relation with cholangiocarcinoma. Infectious Diseases of Poverty 1: 4.

Qian M et al., 2014. From recognition to practicecommemorating the 140th anniversary of Clonorchis sinensis discovery. Chinese Journal of Parasitology and Parasitic Diseases 32: 247-252. 
Rajshekhar V et al., 2003. Taenia solium taeniosis/cysticercosis in Asia: Epidemiology, impact and issues. Acta Tropica 87: 53-60.

Rawla P and Sharma S, 2021. Trichinella spiralis. 2020 May 30. In: StatPearls [Internet]. Treasure Island (FL): StatPearls Publishing; 2021 Jan. PMID: 30860746.

Robert-Gangneux F and Dardé ML, 2012. Epidemiology of and diagnostic strategies for Toxoplasmosis. Clinical Microbiology Reviews 25: 264-296.

Robertson LJ and Chalmers RM, 2013 Foodborne Cryptosporidiosis: Is there really more in Nordic countries? Trends in Parasitology 29: 3-9.

Rochelle PA and Giovanni G, 2014. Cryptosporidium Oocysts in Drinking Water and Recreational Water. Springer Vienna 489-513.

Rollinson D et al., 2013. Time to set the agenda for Schistosomiasis elimination. Acta Tropica 128: 423-440.

Romig T, 2003. Epidemiology of Echinococcosis. Langenbecks Archives of Surgery 388: 209-217.

Roy B et al., 2009. Ultrastructural observations on Fasciolopsis buski and its alterations caused by shoot extract of Alpinia nigra. Microscopy Research Technique 72: 61-66.

Siles-Lucas M et al., 2021. Fascioliasis and Fasciolopsiasis: Current knowledge and future trends. Research in Veterinary Science 134: 27-35.

Sithithaworn P et al., 2012. The current status of Opisthorchiasis and Clonorchiasis in the Mekong Basin. Parasitology International 61: 10-16.

Shimoni Z and Froom P, 2015. Uncertainties in diagnosis, treatment and prevention of Trichinellosis. Expert Review of Anti-infective Therapy 13: 1279-1288.

Sunnotel $\mathrm{O}$ et al., 2006. Cryptosporidium. Letters in Applied Microbiology 43: 7-16.

Tamarozzi F et al., 2020. Epidemiological distribution of Echinococcus granulosus s.l. infection in human and domestic animal hosts in European Mediterranean and Balkan countries: A systematic review. PLoS Neglected Tropical Diseases 14: eooo8519.

Tang ZL et al., 2016. Current status and perspectives of Clonorchis sinensis and Clonorchiasis: Epidemiology, pathogenesis, omics, prevention and control. Infectious Diseases of Poverty 71: 1-12.

Traub RJ et al., 2009. A new PCR-based approach indicates the range of Clonorchis sinensis now extends to Central Thailand. PLoS Neglected Tropical Diseases 3: 367.

Tyzzer EE, 1912. Cryptosporidium parvum (sp. nov.), a coccidium found in the small intestine of the common mouse. Arch Fur Protistenkunde 26: 394418.

Weng YL et al., 1989. Studies on ecology of Fasciolopsis buski and control strategy of Fasciolopsiasis. Zhongguo Ji Sheng Chong Xue Yu Ji Sheng Chong Bing Za Zhi 7: 108-111.

Wiwanitkit V et al., 2002. High prevalence of Fasciolopsis buski in an endemic area of liver fluke infection in Thailand. Medgenmed 4: 6.

Woolsey ID and Miller AL, 2020. Echinococcus granulosus sensu lato and Echinococcus multilocularis: A review. Research in Veterinary Science. 135: 517-522.

$\mathrm{Wu} \mathrm{X}$ et al., 2020. Case report: Surgical intervention for Fasciolopsis buski infection: A literature review. The American Journal of Tropical Medicine and Hygiene 103: 2282-2287.

Yeh HY and Mitchell PD, 2016. Ancient human parasites in ethnic chinese populations. Korean Journal of Parasitology 54: 565-572.

Zhang D et al., 2018. Proteomic profiling of human decidual immune proteins during Toxoplasma gondii infection. Journal of Proteomics 30: 186:28-37. 